\section{Early Access in Oncology: Why Is It Needed?}

\author{
Giovanni Apolone', Andrea Ardizzoni², Giuliano Buzzetti ${ }^{3}$ (D), \\ Mario Alberto Clerico ${ }^{4}$, Pierfranco Conte ${ }^{5}$, Filippo de Braud', \\ Francesco De Lorenzo ${ }^{6}$, Maria Gabriella Ferrandina ${ }^{7}$, \\ Armando Genazzani ${ }^{8}$, Stefania Gori', Michele Maio ${ }^{10}$, \\ Mauro Patroncini ' ', Francesco Perrone ${ }^{12}$, Giovanni Scambia ${ }^{7}$ \\ and Giovanna Scroccaro ${ }^{13}$
}

Global \& Regional Health Technology Assessment

Volume 2019: 1-7

(C) The Author(s) 2019

Article reuse guidelines: sagepub.com/journals-permissions DOI: | 0.1 | 77/22842403| 9857072 journals.sagepub.com/home/grh (3)SAGE

\begin{abstract}
Timely access to cancer therapies with significant added value is an important expectation for patients and a primary responsibility for every public health service. Over time, collaboration between the pharmaceutical industry and regulatory agencies has made it possible to agree to implement tools in order to accelerate the development and approval of potentially innovative drugs.

In Italy, too, several early access tools have been introduced. In June 2018 a panel of experts agreed on the need to simplify and streamline early access assessment criteria and processes.

The panel developed a proposal to categorize cancer drugs eligible for early access. In the curative setting, the evaluation of the medical need should take into account both the relapse rate, attributed on the basis of the disease free survival (DFS), and the strength of the recommendations of the Italian Association of Medical Oncology (AIOM) for any therapeutic alternatives already available. The panel then found it appropriate to use the European Society for Medical Oncology (ESMO) criteria for the evaluation of the clinical benefit. The sum of the scores assigned to the three parameters should allow the clinical value of the drug to be defined and, consequently, the priorities for early access to be established. This multiparameter approach can also be adapted to the non-curative setting.

The early access process should be reserved for first-in-class drugs and should provide for the recognition of a conditional reimbursement within 60 days, financed by a special fund.

The proposal developed by the panel has the objective of starting a proactive discussion with the Italian health authority.
\end{abstract}

\title{
Keywords
}

Cancer drugs, early access, price and reimbursement, clinical value

Date received: 16 April 2019; accepted: 22 May 2019

\footnotetext{
'Fondazione IRCCS Istituto Nazionale dei Tumori, Milano, Italia 2Policlinico Sant'Orsola-Malpighi, Bologna, Italia

${ }^{3}$ Consulta Prevenzione Rischio Cardiovascolare, Dephaforum S.r.I., Milano, Italia

${ }^{4}$ Presidente CIPOMO

${ }^{5}$ Rete Oncologica Veneta, Padova, Italia

${ }^{6}$ European Cancer Patient Coalition, Bruxelles, Belgio

${ }^{7}$ Fondazione Policlinico Universitario A. Gemelli, Roma, Italia

${ }^{8}$ Università degli Studi del Piemonte Orientale, Novara, Italia

${ }^{9}$ Presidente AIOM, Ospedale Sacro Cuore Don Calabria, Negrar

(Verona), Italia
}

\author{
${ }^{10}$ Azienda Ospedaliera Universitaria Senese, Siena, Italia \\ "Dephaforum S.r.I., Milano, Italia \\ ${ }^{12}$ Istituto Nazionale Tumori IRCCS “Fondazione G. Pascale”, Napoli, \\ Italia \\ ${ }^{13}$ Direzione Farmaci, Dispositivi medici e Protesica, Area Sanità e \\ Sociale, Regione Veneto, Venezia, Italia
}

Corresponding author:

Giuliano Buzzetti, Consulta Prevenzione Rischio Cardiovascolare, Dephaforum S.r.I., Milano, Via Cosimo del Fante, 8, Milano, 20122, Italy. Email: g.buzzetti@dephaforum.it 


\section{Introduzione}

I progressi in ambito oncologico osservati negli ultimi anni sono la conseguenza di molteplici fattori tra cui: lo sviluppo di programmi di screening - che consentono una diagnosi precoce - e la realizzazione di percorsi diagnosticoterapeutici (PDTA) che includono una stadiazione accurata e una precisa caratterizzazione molecolare per l'impiego appropriato delle terapie a bersaglio molecolare e dell'immunoterapia.

Queste nuove terapie stanno permettendo sia di ridurre la mortalità nelle fasi precoci di molte neoplasie sia di consentire una cronicizzazione di altre in fase avanzata, a fronte di una buona qualità di vita dei pazienti, legata a una migliore tollerabilità.

Permettere un accesso precoce alle terapie con significativo valore aggiunto, rispetto alle alternative disponibili, rappresenta pertanto una necessità importante per i pazienti oncologici e per i servizi sanitari pubblici.

La collaborazione tra industria farmaceutica e agenzie regolatorie internazionali ha permesso ad oggi di sviluppare e attuare:

- modelli accelerati di ricerca e sviluppo (R\&S) di nuovi farmaci, come il PRIority MEdicine scheme (PRIME); ${ }^{1}$

- percorsi registrativi dedicati, quali:

Accelerated Assessment, ${ }^{2}$ Adaptive Pathways ${ }^{3}$ e Conditional Approval ${ }^{4}$ in Europa e Fast Track, Breakthrough Therapy, Accelerated Approval e Priority Review negli USA. ${ }^{5}$

L'esigenza di un accesso precoce va peraltro pesata attentamente rispetto alle conoscenze sul profilo rischio/ beneficio disponibili al momento del lancio del farmaco. Sono infatti frequenti i casi di autorizzazione EMA in assenza di studi registrativi sufficientemente solidi, lasciando così persistenti incertezze sui rischi associati ai nuovi farmaci, che ricevono spesso l'approvazione, condizionata alla produzione di nuove evidenze sul piano della sicurezza, attraverso i post-authorisation safety studies (PASS).

In Italia si rileva la necessità di ridurre la finestra temporale tra l'approvazione EMA e l'effettivo accesso dei pazienti alle nuove terapie. Tempi di accesso talora dilazionati fino a due anni dall'approvazione $\mathrm{EMA}^{6}-$ per farmaci con un chiaro valore terapeutico aggiunto sono infatti spesso incompatibili con la durata media di sopravvivenza del paziente oncologico con malattia metastatica.

È pertanto ampiamente condivisa la convinzione che sia opportuno rivedere la governance dell'early access, limitatamente ai farmaci con valore terapeutico aggiunto, e affrontare alcune questioni ancora aperte a livello istituzionale, ovvero:
- quali criteri di selezione dei farmaci adottare e chi deve valutare;

- quale processo potrebbe attuare l'Agenzia Italiana del Farmaco (AIFA) per combinare fast track valutativi senza rinunciare al ruolo di approfondimento, di vigilanza e di indirizzo che l'agenzia svolge per promuovere l'appropriatezza prescrittiva e la sostenibilità del sistema;

- come rendere sostenibile la fase di pre-rimborso per tali farmaci;

- come informare correttamente i pazienti della disponibilità di opzioni terapeutiche innovative (si segnala che questo aspetto non è stato preso in considerazione nel presente documento).

Per discutere e proporre possibili soluzioni alle questioni di cui sopra si è costituito un panel interdisciplinare di esperti, che si è riunito in un seminario residenziale il $28 \mathrm{e}$ 29 giugno 2018, per approfondire e finalizzare il lavoro di un precedente incontro di brainstorming propositivo tenutosi nell'ottobre 2017.

Il panel era costituito da oncologi medici e rappresentanti delle istituzioni (nazionali e regionali), delle aziende produttrici, delle associazioni di pazienti e dei media.

\section{La situazione oggi in Italia: quali spazi per accelerare l'accesso ai farmaci}

I nuovi farmaci oncologici vengono valutati con procedura regolatoria centralizzata a livello europeo. In questa fase vengono valutate e definite l'efficacia, la sicurezza e la qualità del farmaco.

La valutazione di EMA però non considera il beneficio incrementale del nuovo farmaco rispetto alle alternative terapeutiche eventualmente già disponibili e non ne valuta l'innovatività. Queste valutazioni sono invece prerogative della fase nazionale, che definisce il reale posizionamento nella terapia (place in therapy) del nuovo farmaco nel contesto, il rapporto incrementale di costo-efficacia e la sostenibilità per il servizio sanitario.

Le difficoltà di contrattare le condizioni di prezzo e rimborsabilità sono principalmente dovute $\mathrm{a}$ :

- prove limitate (o mancanti) di beneficio clinico incrementale del nuovo farmaco rispetto allo standard di cura;

- la complessità, se non impossibilità, di stimare compiutamente il rapporto costo-efficacia;

- la difficoltà di determinare i costi sanitari (diretti e non) e i costi legati alla perdita di produttività, in relazione alla rimborsabilità del farmaco.

L'entità di questi ostacoli sembra purtroppo destinata ad aumentare, data la crescente tendenza ad approvare farmaci sulla base di studi non comparativi, condotti su 
casistiche limitate di pazienti (con caratteristiche specifiche) e con follow-up brevi.

Queste situazioni conducono a un processo negoziale complesso, che può ritardare l'accesso al mercato (delayed access).

Le ripercussioni negative sono importanti. Principalmente vanno considerate e gestite:

- le aspettative dei pazienti, che richiedono la tempestiva disponibilità dei farmaci innovativi, specialmente se questi hanno già ottenuto l'approvazione delle agenzie regolatorie internazionali;

- le aspettative dei clinici di poter disporre di nuove opzioni di cura in patologie senza valide alternative terapeutiche;

- la risonanza mediatica e il rischio di messaggi confondenti tra l'approvazione di EMA e la rimborsabilità in Italia;

- le esigenze delle aziende farmaceutiche, che auspicano di poter operare in un mercato caratterizzato da chiarezza delle regole, trasparenza nelle dinamiche di accesso al mercato, programmazione ed efficienza del sistema.

Strumenti di accesso precoce oggi disponibili in Italia. In Italia l'accesso precoce al mercato per i farmaci meritevoli di tale trattamento e con determinati requisiti può essere realizzato grazie ad alcune norme ad hoc, che fungono da strumenti di accesso. Tale accesso anticipato risulta in parte coperto dallo Stato (Legge 648/96, Legge 326/2003, Legge 94/98) e in parte dall'industria.

La Legge 648/96 prevede, in caso di patologie per cui non sia ancora disponibile alcuna valida terapia, su parere positivo della Commissione Tecnico Scientifica (CTS), l'erogazione a carico del SSN di farmaci: a) innovativi già presenti in altri Stati ma non ancora autorizzati in Italia; b) non ancora autorizzati e sottoposti a sperimentazione clinica; c) disponibili ma approvati in indicazioni diverse. Più recentemente (Legge 79/2014), è stata estesa l'applicazione di questa norma anche in presenza di una valida alternativa terapeutica, consentendo per ragioni di economicità il rimborso di un farmaco in un'indicazione documentata da studi internazionali, seppur differente da quelle approvate dall'agenzia regolatoria. ${ }^{7}$

La legge 648/96 consente un accesso rapido su coorti di pazienti. Tuttavia, difficilmente è associata alla produzione di ulteriori dati sul nuovo farmaco (anche se la normativa lo prevederebbe), non sempre si realizza con una negoziazione formale di prezzo, ha durata protratta fino all'AIC e non si associa a un monitoraggio efficace della spesa (nonostante l'obbligo di rendicontazione trimestrale a carico delle Regioni).

La Legge 326/2003 ha introdotto un fondo AIFA, sostenuto da una tassa del 5\% sulle spese promozionali dell'industria farmaceutica, dedicato per il $50 \%$ al rimborso su base nominale di farmaci orfani per il trattamento di malattie rare e di farmaci che rappresentano una speranza di cura per particolari e gravi patologie. Tale strumento è attualmente poco usato e i relativi fondi allocati solo parzialmente utilizzati: nel 2017 sono stati erogati $€ 13,5$ milioni $^{8}$ (su una disponibilità di circa $€ 17,8$ milioni).

Il D.M. 7/9/2017: disciplina 1'"uso compassionevole", prevedendo che le aziende farmaceutiche possano fornire gratuitamente il farmaco soggetto a sperimentazione clinica ma non ancora autorizzato all'immissione in commercio, in presenza di prove sufficienti di efficacia e tollerabilità.

L'accesso allargato (Expanded Access) è uno studio clinico sponsorizzato dall'azienda farmaceutica per confermare soprattutto il profilo di sicurezza del nuovo farmaco prima della sua commercializzazione, spesso richiesto dall'EMA (PASS.) $)^{8}$

La Legge 94/98 disciplina l'impiego del farmaco nel singolo paziente per indicazioni terapeutiche e modalità di somministrazione off-label, con rimborso da parte delle Regioni.

Nell'ambito degli strumenti specifici di accesso accelerato oggi disponibili in Italia, si possono considerare, anche se di natura diversa dai precedenti, queste ulteriori disposizioni legislative, che seguono la pubblicazione in Gazzetta Ufficiale Europea.

Legge 189/2012 - C(nn): un nuovo farmaco, dopo sessanta giorni dall'autorizzazione EMA, può essere commercializzato e viene inserito in classe C "non negoziata". Istituita per consentire la commercializzazione di farmaci non ancora rimborsati in accordo ai tempi previsti dalla normativa europea, la $\mathrm{C}(\mathrm{nn})$ lascia però l'onere della decisione sull'acquisto del farmaco a livello locale, non essendovi un fondo specifico nazionale per la copertura di tali acquisti. La spesa è pertanto a carico del cittadino o delle singole strutture (ospedali o ASL). Ne consegue il rischio di una frammentazione del sistema e di una differenziazione nell'accesso.

Spesso le aziende farmaceutiche mettono a disposizione il farmaco a un prezzo simbolico in attesa della conclusione della negoziazione di prezzo e rimborso. Inoltre, per i farmaci in $\mathrm{C}(\mathrm{nn})$ non è prevista/richiesta la raccolta di dati di impiego nella pratica clinica (relative effectiveness).

Legge 98/2013 - Nota come "Procedura dei 100 giorni", stabilisce che l'iter negoziale per farmaci orfani, di eccezionale rilevanza terapeutica e sociale e quelli ospedalieri debba avere carattere prioritario. Tuttavia, le associazioni di pazienti e le aziende farmaceutiche evidenziano come il processo sia ancora da ottimizzare e da rendere più trasparente, perché AIFA non rende note le motivazioni per l'ammissione o il rifiuto di tale procedura.

Se è vero che per l'accesso precoce sono disponibili e attuate alcune norme (come la 648 e la 326), è anche vero che gli strumenti oggi disponibili non sono sufficienti per garantire una gestione sistemica e ordinata dell'accesso e 
che gli stessi si prestano a interpretazioni talora soggettive e discrezionali della normativa. Inoltre, si dovrebbe sempre garantire un meccanismo di raccolta dei dati strutturato (Registri), i cui risultati siano pubblicati e valutati nel contesto stesso della negoziazione di prezzo e rimborso oppure successivamente, in fase di rivalutazione.

In sintesi, si ritiene che ci sia una certa ridondanza di strumenti, spesso non coordinati tra loro, e quindi la necessità di una semplificazione e ottimizzazione del sistema, con particolare riferimento alla chiarezza e condivisione dei criteri utilizzati.

\section{II modello francese}

In questo contesto, il panel di esperti ha valutato anche alcune esperienze di altri Paesi europei, dove l'accesso precoce ai farmaci oncologici innovativi è ben regolamentato e attuato. In particolare, è stata apprezzata l'esperienza francese dell'autorisation temporaire d'utilisation (ATU'). ${ }^{10}$ In caso di malattie rare e gravi, che richiedano una terapia non dilazionabile e per le quali non sia disponibile un appropriato trattamento, l'Agence nationale de sécurité du médicament et des produits de santé (ANSM) - su richiesta dell'azienda farmaceutica può approvare l'impiego di farmaci non ancora autorizzati e al di fuori di sperimentazioni cliniche. L'ATU è per indicazione e può essere attivata su base nominale o di coorte, quando sia atteso un evidente beneficio in uno specifico gruppo di pazienti. L'ATU di coorte è gestita in base a un protocollo, concordato fra ANSM e produttore, per la raccolta di dati di tollerabilità e soprattutto di efficacia del farmaco in condizioni reali di utilizzo. L'ATU di coorte ha durata di un anno, eventualmente rinnovabile.

Il costo del trattamento è sostenuto dal sistema pubblico (casse mutue) attingendo al budget dei "farmaci innovativi" e l'azienda farmaceutica trasmette i dati di spesa. Si interrompe una volta ottenuta l'autorizzazione dell'indicazione e la rimborsabilità viene mantenuta fino alla conclusione della procedura di prezzo e rimborso. In caso di successiva discordanza tra prezzo finale e prezzo in corso di ATU, è previsto un meccanismo ben definito di payback (totale o parziale), così pure in caso di spesa annuale superiore a 30 milioni.

\section{Obiettivi del panel}

Completata l'analisi del contesto attuale, il panel di esperti ha identificato due obiettivi principali:

1. Definizione precisa e condivisa dei criteri di selezione dei farmaci veramente meritevoli di early access, attraverso una valutazione oggettiva e trasparente delle aree con elevato bisogno terapeutico e, contestualmente, del valore aggiunto che un nuovo farmaco può portare per il paziente $\mathrm{e}$ per il servizio sanitario.
2. Revisione dell'attuale procedura di fast track autorizzativo in Italia e proposta di un processo volto a favorire un accesso "facile e veloce" ai farmaci oncologici meritevoli, garantendo la sostenibilità del servizio sanitario e farmaceutico.

\section{Criteri di identificazione dei farmaci meritevoli di early access}

Il panel di esperti propone due tipologie di farmaci:

A. Farmaci già individuati da EMA come eleggibili per una valutazione accelerata (Accelerated Assessment, Adaptive Pathway e Conditional Approval) in quanto ritenuti un'innovazione terapeutica di potenziale impatto sulla salute pubblica dal Committee for Medicinal Products for Human Use (CHMP).

B. Nuovi farmaci la cui quantificazione del "medical need" e "valore aggiunto" sia basata su punteggi acquisiti su più strumenti, riconosciuti come riferimento in ambito oncologico e considerati congiuntamente.

Per quanto riguarda la seconda tipologia di farmaci, i pilastri su cui basare la valutazione congiunta sono i seguenti:

1. i criteri di innovatività attualmente considerati da AIFA relativamente a medical need e valore terapeutico aggiunto (mentre la valutazione relativa alla "qualità delle prove" non è ritenuta appropriata al contesto);

2. le raccomandazioni sull'uso dei farmaci elaborati dall'AIOM;

3. i criteri per la stratificazione del grado di beneficio clinico dei farmaci elaborati dalla European Society for Medical Oncology (ESMO).

Tali criteri vanno applicati in due diversi setting clinici:

- curativo (ove l'obiettivo del trattamento è curativo/ adiuvante);

- non curativo (in casi di malattia in stato molto avanzato).

La metodologia proposta dal panel è di seguito illustrata, come esempio, per il solo setting curativo. Lo stesso metodo, con punteggi ovviamente rivalutati in funzione della severità della situazione, è proposto anche per il setting non curativo.

\section{Setting curativo}

Valutazione del medical need. Vanno considerati due parametri: 
Tabella I. Punteggio relativo al bisogno terapeutico.

\begin{tabular}{lll}
\hline Tasso di ricadute & Bisogno terapeutico & Punteggio per early access \\
\hline Basso $<10 \%$ & Basso & 1 \\
Intermedio $10 \%-30 \%$ & Intermedio & 3 \\
Alto $>30 \%$ & Alto & 5 \\
\hline
\end{tabular}

Tabella 2. Punteggio correlato alle raccomandazioni di AIOM.*

\begin{tabular}{ll}
\hline Forza delle raccomandazioni delle linee guida AIOM & $\begin{array}{c}\text { Punteggio } \\
\text { per early access }\end{array}$ \\
\hline Positiva forte relativa ad alternative terapeutiche disponibili e molto efficaci & I \\
Positiva debole per presenza di alternative terapeutiche disponibili, di efficacia moderata & 2 \\
Negativa debole per presenza di alternative terapeutiche disponibili, di efficacia scarsa & 3 \\
Negativa forte per assenza di alternative disponibili & 4
\end{tabular}

*Es. Se AIOM esprime raccomandazione "Positiva Forte" per un farmaco già disponibile, ne deriva che esistono "alternative terapeutiche disponibili e molto efficaci” e quindi il punteggio da attribuire al nuovo farmaco ai fini dell'early access non potrà essere superiore a I; a raccomandazione AIOM positiva debole, il punteggio per il nuovo farmaco sarà 2; a raccomandazione AIOM negativa debole corrisponde punteggio pari a 3 per early access; raccomandazione AIOM negativa forte equivale a punteggio 4 per early access.

Tabella 3. Punteggio in base ai criteri dell'European Society for Medical Oncology.

\begin{tabular}{ll}
\hline Scala ESMO-MCBS & Punteggio per early access \\
\hline Grado A & 9 \\
Grado B & 6 \\
Grado C & 3 \\
\hline
\end{tabular}

ESMO-MCBS: European Society for Medical Oncology-Magnitude of Clinical Benefit Scale.

A. Il tasso di ricadute.

Il punteggio viene attribuito in base alla sopravvivenza libera da malattia (disease-free survival, DFS), contestualizzato per patologia, studio e tempo.

La Tabella 1 riporta la correlazione tra livello di bisogno terapeutico e punteggio ai fini dell'early access.

B. La presenza di alternative terapeutiche considerate efficaci.

Per il punteggio ai fini dell'early access si fa riferimento alla forza delle raccomandazioni espresse dall'AIOM per stabilire la presenza o meno di alternative valide già disponibili (Tabella 2).

Valutazione del valore aggiunto del farmaco. Il grado di beneficio clinico di un farmaco oncologico è attribuito in base ai criteri ESMO-(ESMO-MCBS.). ${ }^{11,}{ }^{12}$ La Tabella 3 riassume il punteggio assegnato dal gruppo di lavoro per ogni grado di valutazione della scala ESMO.

Valutazione del valore clinico del farmaco. La valutazione globale dei 3 parametri permetterà di ottenere un
Tabella 4. Valore Clinico del Farmaco.

\begin{tabular}{ll}
\hline $\begin{array}{l}\text { Punteggio finale- setting } \\
\text { curativo }\end{array}$ & $\begin{array}{l}\text { Priorità per l'early } \\
\text { access }\end{array}$ \\
\hline Da 5 a 7 & Molto bassa \\
Da 8 a 10 & Bassa \\
Da II a 13 & Intermedia \\
Da 14 a 15 & Alta \\
Da 16 a 18 & Molto alta \\
\hline
\end{tabular}

punteggio complessivo Medical Need + Valore Aggiunto, definito "Valore Clinico del Farmaco" variabile da 5 a 18 , che definisce l'eleggibilità di un nuovo farmaco all'early access (Tabella 4).

Nel setting CURATIVO, il panel raccomanda una soglia per l'early access di almeno 14 punti, ovvero priorità 'alta' o 'molto alta'.

Nel setting NON CURATIVO si adotteranno dei correttivi, essendo il medical need massimo per definizione.

Questa proposta elaborata dal panel di esperti va letta sotto il profilo soprattutto metodologico e qualitativo. Sono quindi necessari approfondimenti e una ulteriore validazione che possa dimostrarne sia la concordanza con i criteri di innovatività di AIFA in tutti $\mathrm{i}$ setting sia l'applicabilità come strumento di valutazione.

Rappresenta comunque una base di partenza innovativa, sicuramente migliorabile, anche in funzione della discussione che seguirà alla presentazione della proposta agli attori coinvolti.

Questo metodo di valutazione quantitativa per l'eleggibilità dei farmaci potrà essere in futuro utilizzato dalle aziende farmaceutiche anche in modo dinamico, per candidare il proprio farmaco all' early access (aggiornando 
il dossier con dati rilevanti che si rendano disponibili successivamente).

AIFA avrà la responsabilità di decidere se effettivamente sussistano le condizioni per cui il farmaco sia meritevole di un percorso accelerato.

\section{Quale fast track autorizzativo per i farmaci meritevoli di accesso precoce?}

Il panel di esperti propone che il percorso di early access debba essere selettivo e riservato esclusivamente ai farmaci (o indicazioni terapeutiche) "first in class", che raccolgano i punteggi di valore clinico del farmaco al di sopra di una certa soglia per consentirne l'eleggibilità.

I farmaci second in class potranno essere meritevoli di early access solo in caso di evidenze scientifiche che ne dimostrino un beneficio clinico aggiuntivo.

Nell'ambito di una nuova governance dell'early access, il panel propone che l'Azienda possa inoltrare alla CTS la domanda di ammissione al percorso di early access immediatamente dopo il parere positivo del CHMP.

Alla CTS viene richiesto il rispetto di una tempistica ben definita per valutare il dossier ed emettere un suo giudizio (60 giorni sembra essere un lasso di tempo ragionevole).

L'accettazione al percorso di early access permetterà all'Azienda di entrare immediatamente sul mercato con farmaco rimborsato dal SSN a un prezzo negoziato in via temporanea - che dovrà fare riferimento ai prezzi medi negoziati negli altri Paesi europei - e con apposito registro di patologia.

Il periodo di early access potrà durare al massimo 12 mesi, durante i quali dovrà aver luogo la procedura di negoziazione di prezzo e rimborsabilità.

Una volta raggiunto l'accordo sul prezzo del farmaco per la rimborsabilità, l'Azienda sarà tenuta a restituire l'eventuale differenza tra il prezzo inizialmente definito per l'early access e quello negoziato.

In caso invece di non rimborsabilità, il periodo di early access termina e il farmaco non potrà rimanere in commercio con la rimborsabilità da parte del SSN. Sarà comunque assicurata la continuità terapeutica per i pazienti che hanno utilizzato il farmaco durante il periodo di early access. L'azienda farmaceutica provvederà inoltre al rimborso della spesa sostenuta in precedenza dal SSN durante il periodo di early access in base a criteri da definire.

Finanziamento. Relativamente alla sostenibilità dell'early access, il panel ne propone il finanziamento attraverso un fondo dedicato. Il fondo per l'early access potrà anche essere alimentato da:

- quota non spesa del fondo 5\% della Legge 326;
- rimborsi delle Aziende derivanti dalla eventuale differenza tra il prezzo inizialmente fissato per l'early access e quello negoziato;

- pay back della spesa sostenuta dal SSN in caso di non rimborsabilità.

La possibilità di finanziare l'early access con un fondo nazionale dedicato e con un modello auto sostenibile eliminerà la necessità di ulteriori valutazioni a livello regionale, che altrimenti rischierebbero di rallentare l'accesso ai farmaci, determinando anche una diseguaglianza territoriale tra i pazienti.

Qualora l'Azienda, oltre all'ammissione al percorso di early access, abbia anche fatto richiesta di riconoscimento di innovazione terapeutica e il giudizio finale di AIFA sia stato positivo (innovatività importante), il farmaco uscirà dalla fase di early access e potrà accedere a pieno titolo ai fondi per i farmaci oncologici innovativi.

\section{Discussione}

Il panel, consapevole che la proposta delineata dovrà essere discussa e approfondita con tutti gli attori coinvolti, auspica che il documento possa essere recepito come una base solida per un confronto franco sull'urgenza di trovare una soluzione al reale bisogno dei pazienti evidenziato in questo documento: avere accesso velocemente e facilmente a terapie innovative e con un chiaro valore aggiunto, laddove la malattia non permetta ritardi.

Un'ulteriore opportunità offerta dai 12 mesi di early access è l'implementazione di registri di patologia e la raccolta di evidenze aggiuntive relative all'utilizzo del farmaco in un contesto di reale pratica clinica. Tenuto conto della (sempre più) frequente approvazione in sede europea di farmaci con documentazione non ancora completa e soggetti all'obbligo di studio confirmatorio, la raccolta di dati di real world evidence (RWE) può risultare di cruciale utilità per le autorità regolatorie nazionali, soprattutto se questa attività, che offrirà un significativo contributo all'incremento delle conoscenze, verrà appropriatamente normata nell'interesse del SSN.

A tal fine il panel suggerisce di proporre a Governo e Parlamento l'emendamento di uno strumento legislativo già esistente, ovvero la Legge 648, che, se rivista in base alle proposte del panel, potrebbe realmente diventare il percorso normativo di early access da implementare in situazioni di alto "medical need" e per farmaci ad alto "valore aggiunto".

\section{Declaration of Conflicting Interest}

The authors declared no potential conflicts of interest with respect to the research, authorship and/or publication of this article. 


\section{Funding}

This article is the result of collegial work performed during the Seminario sull'early access in oncologia, held on June 28 and 29, 2018 at Villa Braida, Mogliano Veneto, Treviso, Italy, with unconditional support from the following consortium of companies: Amgen, Astrazeneca, BMS, Celgene, Ipsen, Janssen, Lilly, MSD, Novartis, Pfizer, Sanofi and Tesaro.

Dephaforum S.r.l., which organised the seminar, paid an educational fee to: AA, PC, FDB, SG, MM, MP and FP. None of the other authors - GA, GB, AC, FDL, GF, AG, GSca and GScr - received any sort of payment.

\section{ORCID iD}

Giuliano Buzzetti iD https://orcid.org/0000-0002-4197-9871

\section{Bibliografia}

1. European Medicines Agency (EMA). Two years of PRIME, http://www.ema.europa.eu/ema/index.jsp?curl=pages /news_and_events/news/2018/05/news_detail_002953 .jsp\&mid $=\bar{W} C 0 \mathrm{~b} 01 \mathrm{ac} 058004 \mathrm{~d} 5 \mathrm{c} 1$ (2018, accessed 2 June 2019).

2. European Medicines Agency (EMA). Accelerated assessment, http://www.ema.europa.eu/ema/index.jsp?curl=pages/regulation /general/general_content_000955.jsp\&mid=WC0b01ac 05809f843a (2011, accessed 2 June 2019).

3. European Medicines Agency (EMA) Adaptive pathways, http://www.ema.europa.eu/ema/index.jsp?curl=pages/ regulation/general/general_content_000601.jsp\& mid=WC0b01ac05807d58ce (2015, accessed 2 June 2019).

4. European Medicines Agency (EMA). Conditional marketing authorisation, http://www.ema.europa.eu/ema /index.jsp?curl=pages/regulation/general/general_content _000925.jsp (2018, accessed 2 June 2019).
5. US Food \& Drug Administration (FDA). Fast Track, Breakthrough Therapy, Accelerated Approval, Priority Review, https://www.fda.gov/forpatients/approvals/fast/default.htm (2018, accessed 2 June 2019).

6. Lidonnici D, Ronco V, Isernia M, et al. Temi di accesso ai farmaci in Italia nel periodo 2015-2017: Analisi delle tempistiche di valutazione dell'Agenzia Italiana del Farmaco. Global \& Regional Health Technology Assessment 2018: 1-9.

7. Agenzia Italiana dei Farmaci (AIFA). Legge 648/96, www .agenziafarmaco.gov.it/content/legge-64896 (2019, accessed 2 June 2019).

8. Osservatorio Nazionale sull'Impiego dei Medicinali O. L'uso dei farmaci in Italia. Rapporto nazionale 2017. Rome: Agenzia Italiana dei Farmaci, 2018, p. 89.

9. European Medicines Agency (EMA). Post-authorisation safety studies (PASS). https://www.ema.europa.eu/en /human-regulatory/post-authorisation/pharmacovigilance /post-authorisation-safety-studies-pass-0 (accessed 2 June 2019).

10. Agence nationale de sécurité du médicament et des produits de santé (ANSM). Notice to applicants for marketing for Temporary Authorisation for Use (ATU). https://ansm. sante.fr/var/ansm_site/storage/original/application/cadfbcf 9594614d59c8915670853a28b.pdf (2015, accessed 2 June 2019).

11. Cherny N, Sullivan R, Dafni U, et al. A standardised, generic, validated approach to stratify the magnitude of clinical benefit that can be anticipated from anti-cancer therapies: The European Society for Medical Oncology Magnitude of Clinical Benefit Scale (ESMO-MCBS). Ann Oncol 2015; 26: 1547-1573.

12. Cherny NI, Dafni U, Bogaerts J, et al. ESMO-Magnitude of Clinical Benefit Scale version 1.1. Ann Oncol 2017; 28: 2340-2366. 2017/09/26. 